\title{
THE NUCLEATION OF RECRYSTALLISATION AND THE DEVELOPMENT OF TEXTURES IN HEAVILY COMPRESSED IRON-CARBON ALLOYS
}

\author{
I. L. DILLAMORE, H. KATOH $\dagger$ and K. HASLAM \\ British Steel Corporation, Corporate Laboratories, Sheffield, U.K.
}

(Received May 2, 1972; revised June 22, 1972)

\begin{abstract}
Quantitative studies of textures developed in compressed iron- $0.02 \% \mathrm{C}$ and iron- $0.14 \% \mathrm{C}$ alloys before and after annealing have allowed theoretical predictions based on homogeneous plasticity theory to be assessed. It is concluded that qualitatively the theory is very good but predicts rates of texture development rather higher than those observed. The prediction of the theory that orientations on a line joining [110] and [411] should have a tendency to split into two components on deformation and form transition bands, which may be important in nucleating recrystallisation, has been confirmed by the results of the annealing experiments.
\end{abstract}

\section{INTRODUCTION}

The development of fibre textures has received very little attention compared with the more commonly investigated rolling textures. The reason for this is that fibre textures are of limited commercial importance but from the experimental and theoretical viewpoint they have two major advantages. It is a relatively easy matter to obtain fully quanțitative textural information for axisymmetric textures and the relationship between deformation and annealing textures is more readily discerned than for rolling textures. The mechanisms of texture development, both in deformation and on annealing are basically similar for all deformation geometries so it is possible to take advantage of the simplified approach afforded by the axisymmetric situation to obtain information of a more general character.

The present work arose out of a general study of the mechanisms of recrystallization after large strain deformation, a particular interest being in the development of microstructural heterogeneities during deformation and their importance in recrystallisation. It was recognised ${ }^{1}$ that in rolling deformation a number of orientations, notably $\{110\}\langle 001\rangle$ in b.c.c. metals and $\{100\}\langle 001\rangle$ in f.c.c. metals, have a high probability of splitting into two or more components linked by a transition

†Nippon Steel Corporation, Kitakyushu, Japan-formerly on secondment to British Steel Corporation, Corporate Laboratories, Sheffield. band, with a small residue of the initial orientation remaining in the transition region. These orientations form strong components of the recrystallisation textures in metals of the two crystal structures and it is fairly well established ${ }^{2}$ that this is because the geometry of the transition band makes it a favourable site for the nucleation of recrystallisation.

Similar orientation splitting can occur in axisymmetric deformation but the simple one axis situation allows another principle to be recognised. This can be demonstrated by Figure 1, which shows the calculated trends of slip rotations for b.c.c. metals deforming homogeneously by pencil glide with an imposed axisymmetric strain. In compression the slip rotations are towards either [111] or [100], in tension they are towards [110]. The dashed line in Figure 1 linking [110] to [411] is important in the present context. In compression a grain whose axis orientation lies on this line rotates towards [411] but if it were to get displaced accidentally to one side of the line it would continue to rotate away from the line. This line will be termed a divergent line for the compression case; it would be a convergent line in tension. The [011] zone is a convergent line in compression and a divergent line in tension.

It is very likely that a grain on or close to the [110]-[411] divergent line could become bent across the line so that one part of the grain rotates towards [100] and the other to [111]. The progress with strain of three orientations which receive a $1^{\circ}$ 


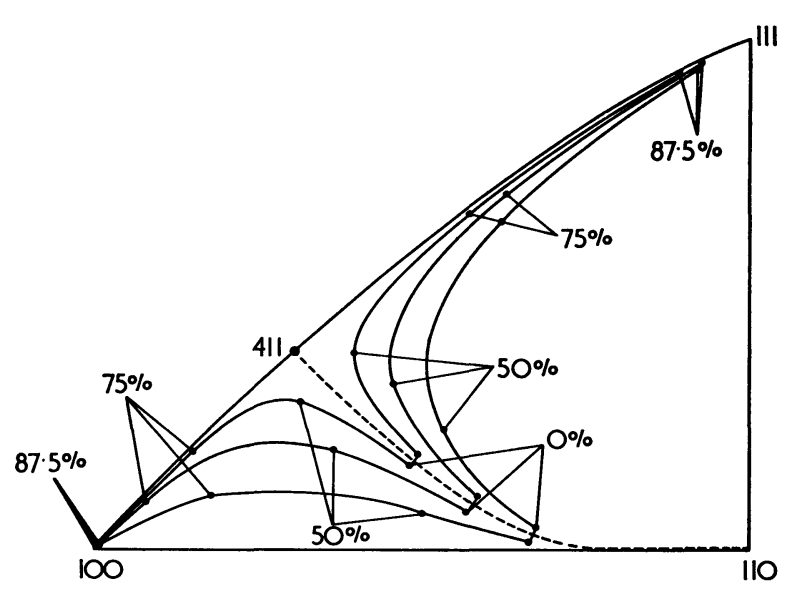

FIGURE 1 Slip rotation paths for axisymmetric deformation of b.c.c. metals deforming by pencil glide.

displacement towards either side of the line is charted in Figure 1. The lattice curvature generated in producing these displacements naturally bridges the initial orientation and as straining proceeds the orientation range will spread progressively because of the divergence, but the small residue of the initial orientation will continue to rotate towards [411].

Considering only the [411] orientation, this will split by rotation in opposite senses along the [011] zone and be present after deformation as a small residue in a transition band. On the principles stated earlier it may thus be expected that recrystallisation nuclei will be present having an orientation close to [411]. However, the initial volume fraction of grains close in orientation to [411] is very small, so these nuclei would be few in number if it were not for the fact that the transition bands formed all the way along the [411]-[110] line rotate to bring the overall curvature in line with the [011] zone. This accumulation should make a substantial number of nuclei of near [411] orientation available and it would thus be expected that the recrystallisation texture should contain material in this orientation. In the following this notion has been examined by compressing samples of iron and examining the deformation and annealing textures.

\section{EXPERIMENTAL METHODS}

Two samples of material were used in the work, both were vacuum melted from a Swedish iron base containing low residuals, one contained $0.02 \%$ carbon, the other $0.14 \%$ carbon, there were no other deliberate additions. Specimens were pro- duced from hot rolled rods of the two materials in the form of cylinders $17 \mathrm{~mm}$ high and $12.7 \mathrm{~mm}$ in diameter. In the starting condition the lower carbon material had a grain size of about $100 \mu \mathrm{m}$ compared with $20 \mu \mathrm{m}$ for the higher carbon material. Both materials were randomly oriented. The cylinders were deformed incrementally in compression, with teflon lubrication being re-applied between each increment, total deformations of both $\sim 70 \%$ and $\sim 85 \%$ were used for the two materials; to achieve the higher deformation it was necessary to reduce the specimen diameter to $\sim 12.7$ $\mathrm{mm}$ after $70 \%$ deformation.

Texture measurements were made on a Siemens texture goniometer with an auxiliary motor to rotate the specimen rapidly about the compression axis. The intensity was determined at 5 degree intervals as the axis was rotated from a position parallel to the reflecting plane normal to an angle of $65^{\circ}$ from the plane of focussing. Three reflections were used (220), (200) and (222) and the intensity data was used following the principles described by Jetter et $a l .,^{3}$ involving matrix inversion, to plot quantitative inverse pole figures. Texture sampling was carried out on the deformed material after grinding, and finally etching, $25 \%$ of the sample from one side only. After determining the deformation texture the samples were annealed, in salt bath for short duration anneals and in a muffle furnace with a flow of hydrogen with a dew point of $20^{\circ} \mathrm{C}$ for long time annealing. The sample for texture analysis was prepared by removing $25 \%$ of the thickness from the side opposite to that used in examining the deformation texture.

\section{RESULTS AND DISCUSSION}

The compression textures for the low-carbon iron after $69.7 \%$ and $87.7 \%$ are shown in Figures $2 a$ and $2 \mathrm{~b}$ respectively. Two conclusions can be drawn from these figures, namely that the [100] texture develops more rapidly than the [111] texture, which is expected from the calculations as evidenced by Figure 1, and the rotations divide about the theoretically derived line shown in Figure 1, with $\sim 70 \%$ rotating towards [111] and $30 \%$ going to [100]. The theoretical division is $77 \%$ [111] and $23 \%$ [100].

The higher carbon material shows substantially similar features to the low-carbon iron with the only difference being that texture development is rather slower for the higher carbon content. This may be seen by comparing the results of Figure 3 with those 


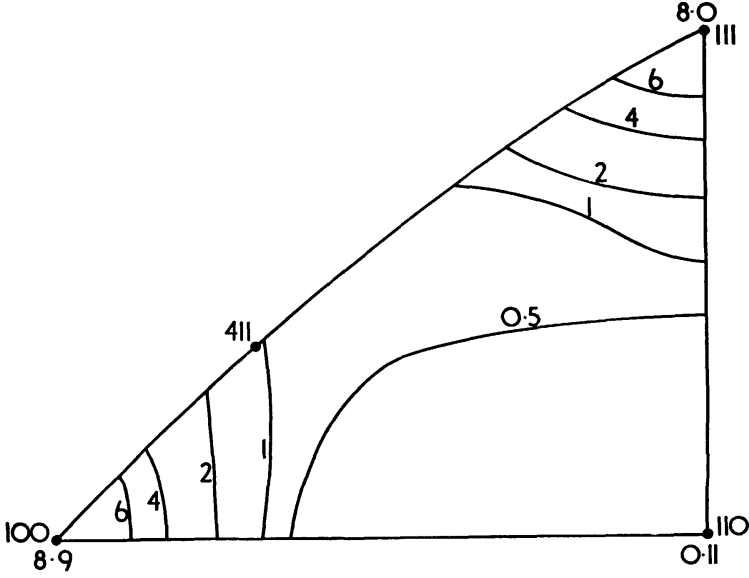

(2a)

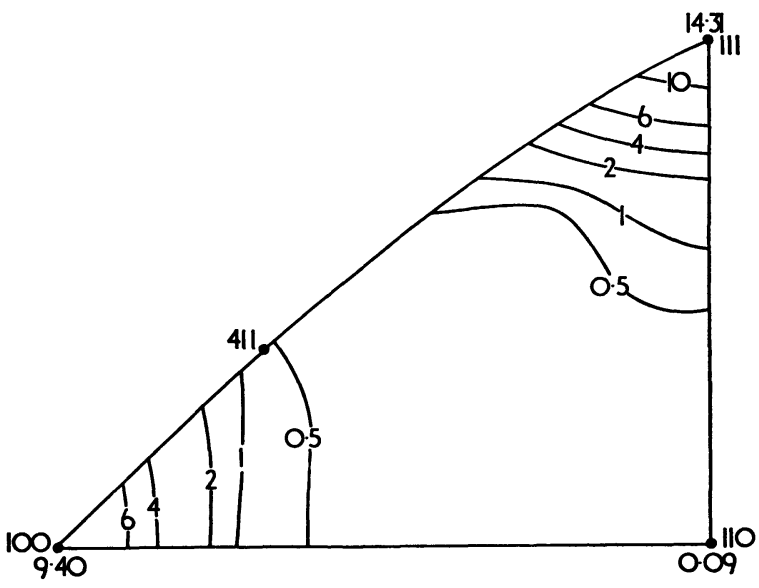

(2b)

FIGURE 2 Compression texture for $\mathrm{Fe}-0.02 \% \mathrm{C}$ alloy (a) deformed 69.7\%; (b) deformed $87.7 \%$.

of Figure 2. It is not perhaps surprising that a material containing $\sim 15 \%$ pearlite should show slower texture development than a pearlite-free material since the inability of the pearlite to deform freely with the cementite imposes additional strain conditions. Both materials show somewhat slower texture development than predicted by homogeneous deformation theory involving pencil glide. The contributory factors to this shortfall are probably that deformation is not truly homogeneous and slip may not be exactly by pencil glide. The theory is not, however, very far from the facts for the low-carbon iron and the results are sufficiently good to feel some confidence in the utility of homogeneous theory for high strain deformation.

Annealing textures developed in the low-carbon iron after $2 \mathrm{~min}$ at $700^{\circ} \mathrm{C}$ in salt bath are shown in Figures $4 \mathrm{a}$ and $4 \mathrm{~b}$. After $70 \%$ deformation the annealing texture produced is rather weak, annealing has produced a near random material (Figure 4a). After $87.7 \%$ deformation and $2 \mathrm{~min}$ at $700^{\circ} \mathrm{C}$ the texture is again weaker after annealing than the deformation texture but the annealing texture is still quite strong. Its main features are a high [111] component and a moderate [411] component. The [111] component is substantially weaker than the

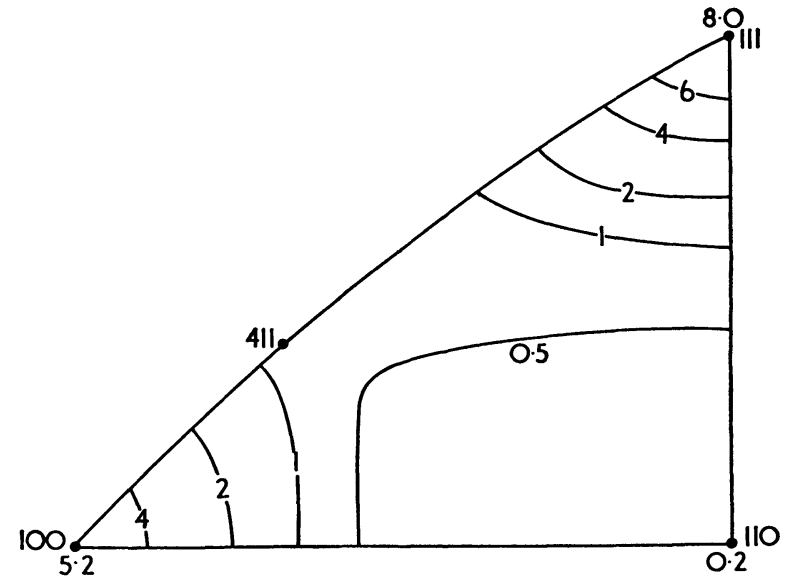

(3a)

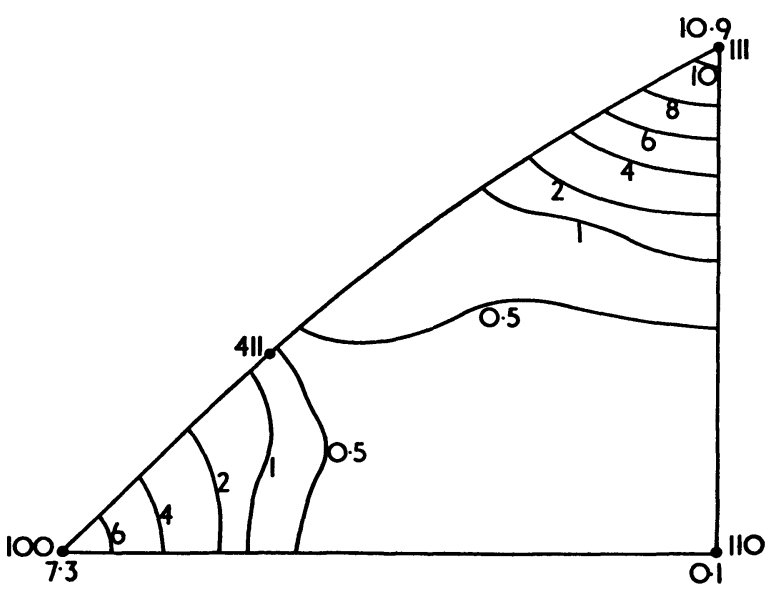

(3b)

FIGURE 3 Compression texture for $\mathrm{Fe}-0.14 \% \mathrm{C}$ alloy: (a) deformed 70\%; (b) deformed $88.5 \%$. 


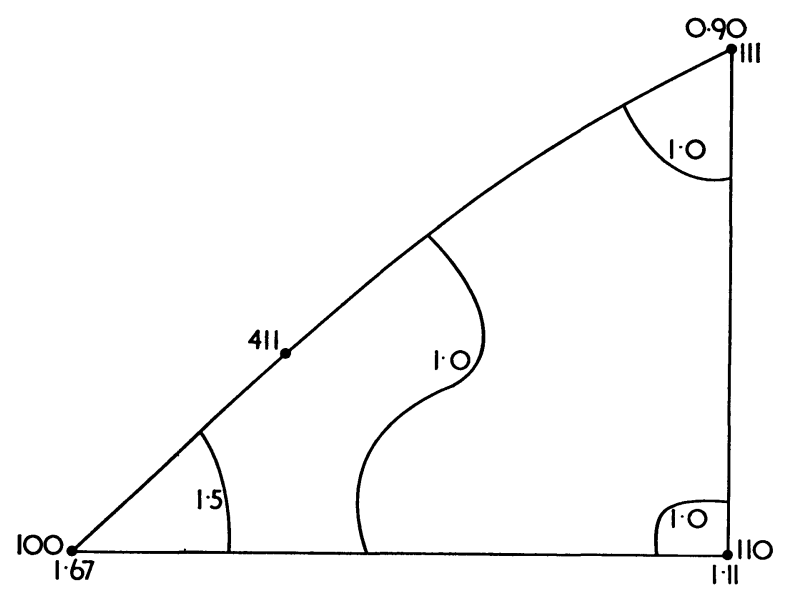

(4a)

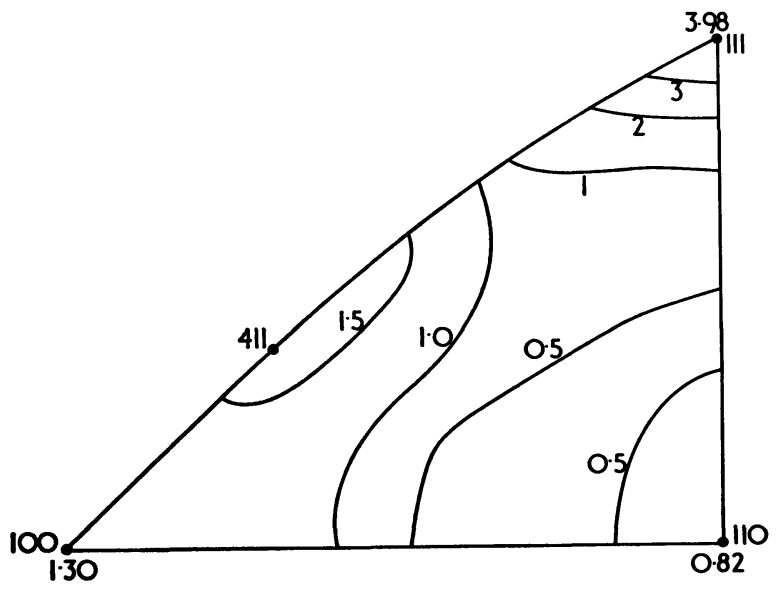

(4b)

FIGURE 4 Annealing textures for $\mathrm{Fe}-0.02 \% \mathrm{C}$ alloy after annealing $2 \mathrm{~min}$ at $700^{\circ} \mathrm{C}$ in salt bath and following: (a) $69.7 \%$ compression; (b) $87.7 \%$ compression.

deformation texture (3.98 compared with 14.31) while the near [411] component is greatly increased by annealing, from 0.28 to 1.60 . The [110] component also shows a strong increase, from 0.09 to 0.82 .

The [111] recrystallised component undoubtedly arises by a subgrain growth mechanism, similar to that operating in (111) planar orientations ${ }^{4}$ in rolled iron, since the dislocation structure for the two cases will be similar. The other components, near [411] and the enhanced [110], are the only ones showing a substantial volume increase on annealing and they must arise from transition band nucleation of the type outlined in the introduction. The [110] orientation splits between [111] and [111] on deforming and for this range of orientation the axis of curvature is [110]. These results provide striking confirmation of the importance of transition bands in providing recrystallisation nuclei.

In the low-carbon iron nucleation of recrystallisation must occur either at pre-existing grain boundaries or within grains. Intragranular nucleation is by subgrain growth, which is most favoured in the two extreme environments of a sharp lattice curvature, as in a transition band, or zero long range lattice curvature. The stable [100] and [111] orientations are expected to show little lattice curvature because they are convergent orientations. Subgrain growth is likely to be more rapid in [111] grains than [100], for reasons which have been discussed previously, ${ }^{4}$ and the [111] orientation will thus be more capable of surviving after recrystallisation.

In the higher carbon iron there is an additional source of recrystallisation nuclei in the presence of the pearlite colonies, coarse second phases being well established as stimulants to nucleation. It is thus of interest to examine the competition between this form of nucleation and the others which have been considered.

A series of annealing experiments were carried out, each for obvious reasons being performed on a different sample. In the first, $2 \mathrm{~min}$ at $700^{\circ} \mathrm{C}$ in salt bath was used for comparison with the low carbon material. The $70 \%$ compressed sample showed a very weak texture and no further work was done on this material. The material compressed $88.5 \%$ gave a texture very similar to that found in the low-carbon material, as shown by Figure 5a, which should be compared with Figure $4 \mathrm{~b}$.

It may be supposed that the components present in the texture of Figure 5a arose from either subgrain growth, transition bands or at second phase particles. A simple experiment to distinguish these components can be performed by raising the temperature so that the original pearlite colonies transform to austenite, grains nucleated near to the pearlite can be annihilated in this process, and if decarburisation is then used to remove the austenite without changing the temperature the grains nucleated further away from the pearlite will be allowed to grow as the austenite-ferrite interface recedes. This simple experiment was performed on a sample annealed for $2 \mathrm{~min}$ at $700^{\circ} \mathrm{C}$ followed by $24 \mathrm{hr}$ at $780^{\circ} \mathrm{C}$ in wet hydrogen. At this temperature about $30 \%$ austenite is formed within a short 


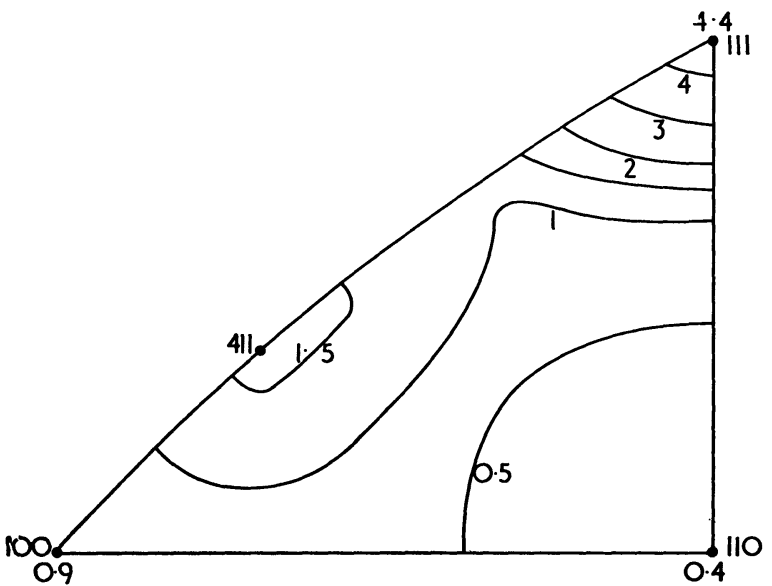

(5a)

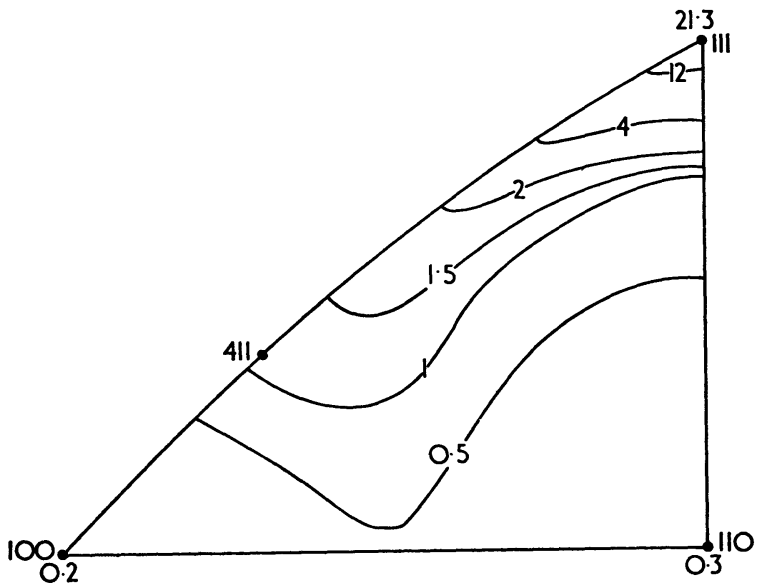

(5b)

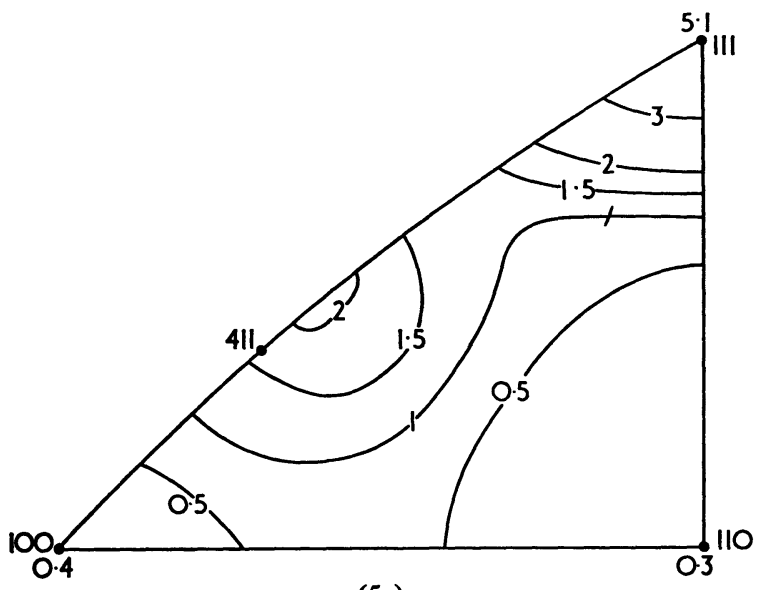

(5c)

FIGURE 5 Annealing textures for $\mathrm{Fe}-0.14 \% \mathrm{C}$ alloy deformed $88.5 \%$ and $(a)$ annealed $2 \mathrm{~min}$ at $700^{\circ} \mathrm{C}$; $(b)$ annealed $2 \mathrm{~min}$ at $700^{\circ} \mathrm{C}$ followed by $24 \mathrm{hr}$ at $780^{\circ} \mathrm{C}$ in wet $\mathrm{H}_{2} ;\left(\right.$ c) annealed $23 \mathrm{hr}$ at $715^{\circ} \mathrm{C}$ in wet $\mathrm{H}_{2}$.

C time of reaching $780^{\circ} \mathrm{C}$ and this is gradually reduced to zero by decarburisation.

The resulting texture is shown in Figure 5b. The prominent feature of this texture is the dramatically increased volume fraction of the [111] component. The [411] component is slightly weakened and the general background is very much weaker. It may be concluded from this that the particle stimulated nucleation is essentially random in orientation and the [111] orientation originates from locations remote from the carbides. This latter conclusion is not surprising if [111] nuclei originate by subgrain growth in regions of essentially uniform orientation, since the second phase particles will perturb the surrounding material. Such perturbation near to particles is likely to promote lattice curvatures leading to transition-band development, so that [411] nuclei will on average be near to but not immediately adjacent to the particles. In the early stages of the high temperature anneal, grains of this orientation will suffer growth restraint due to the nearby austenite grains.

A further experiment using the presence of the carbide phase was carried out to give some information of the relative sizes of the differently oriented grains in the as recrystallised grain size distribution. This technique has been used with success in previous experiments ${ }^{5}$ and uses the fact that if sufficient second phase particles are present to completely inhibit grain growth, when the fraction of second phase is progressively reduced the largest grains in the distribution are the first to grow. Gradually reducing the volume fraction, or allowing particle coarsening to occur, will continue to allow only the largest grains to grow.

In the experiment using this technique a sample was annealed for $24 \mathrm{hr}$ at $715^{\circ} \mathrm{C}$ in wet hydrogen. The second phase restraint of grain growth is first reduced by spheroidisation of the cementite and later the decarburisation will predominate. The texture produced is shown in Figure 5c. The grain growth produced by this treatment was not excessive, the grain diameters after $2 \mathrm{~min}$ at $700^{\circ} \mathrm{C}$ and $23 \mathrm{hr}$ at $715^{\circ} \mathrm{C}$ being $8 \mu \mathrm{m}$ and $13 \mu \mathrm{m}$ respectively. However, there are significant changes between the data of Figures 5a and 5c. The [111] component is increased, but only by $15 \%$, the near [411] component is increased by $30 \%$ and the random background is reduced.

These results suggest that particle induced nuclei are the smallest, nuclei forming by subgrain growth in regions of essentially uniform orientation are of intermediate size and those forming at transition 
bands are on average at the larger end of the size range. These deductions apply only to the present circumstances but are of wider importance.

The nucleation of recrystallisation from transition bands is likely to be markedly dependent upon strain, since at low strains the curvature will not be very well developed and a subgrain will have to grow for a long way before it acquires a high angle boundary, while at high strains the subgrain may develop a high angle boundary too rapidly and become subcritical for nucleation. As indicated by the results of Figures $2 a$ and $4 a$, at $70 \%$ deformation the transition band containing [411] is only just becoming sufficiently curved to act as a nucleation site. The curvature which exists at this strain can be estimated from Figure 1. At the higher strain it is possible that the transition band is past the peak of its effectiveness, but this has not been investigated in detail. Judging by the results of Figures $4 a$ and $4 b$, in the absence of particle interference, the mechanisms competing with transition band nucleation are, at low strain, grain boundary and grain corner nucleation and at high strains subgrain nucleation. At the lower strain used here the [100] component probably originates from grains boundary nucleation as previously discussed for the case of rolled iron. ${ }^{6}$

It should be mentioned finally that the [411] component found in the compression annealing texture of iron (and almost certainly in all other b.c.c. metals) is expected to occur in the tension texture also. In tension rotation will occur from [111] to [411] and from [100] to [411] during deformation and the [411] component will split between [110] and [101], giving a transition band capable of proving recrystallisation nuclei. This remains to be proven and may be complicated by the predilection of grains in the [110] end texture to achieve plane strain conditions involving substantial inhomogeneous deformation. ${ }^{7}$

\section{CONCLUSIONS}

1) The deformation texture in compression of a single phase iron alloy is substantially the same as predicted by homogeneous deformation theory assuming pencil glide. The texture develops rather more slowly and is thus less sharp than the theory predicts. The introduction of a sizeable volume fraction of second phase does not alter the general features of the texture but further slows down the texture development.

2) The overall tendencies of the slip rotations are as predicted by the theory and this leads to the development of lattice curvatures about specific orientations, in compression these orientations lie on a line joining [110] and [411]. The [110] orientation splits in deformation to give [111] and [111] components. Other orientations on the [110]-[411] split between [100] and [111] but rotate so that the curvature joining these orientations lies along the [011] zone. Such a curvature is expected to provide recrystallisation nuclei under certain conditions.

3) It has been confirmed that orientations near [411] arise on recrystallising heavily compressed iron. This is the first report of this orientation being developed, but since it is also the first systematic quantitative study of texture development in axisymmetrically deformed b.c.c. metals this is not surprising.

4) The [411] orientation arises most probably in the way which was predicted, namely by nucleation in regions of lattice curvature formed by orientation splitting.

5) Some experiments involving intercritical annealing and decarburisation have shown that particle stimulated recrystallised grains are smaller than the average and are essentially random in orientation.

\section{REFERENCES}

1. I. L. Dillamore and H. Katoh, in Polish Academy of Sciences Proc. Conf. on Quantitative Analysis of Textures (in press).

2. $\mathrm{H}$. $\mathrm{Hu}$, in Textures in Research and Practice, J. Grewen and G. Wassermann, eds. (Springer, Berlin, 1969), pp. 200-226.

3. L. K. Jetter, C. J. McHargue and R. O. Williams, J. Appl. Phys. 27, 368 (1956).

4. C. J. E. Smith and I. L. Dillamore, Met. Sci. J. 4, 161 (1970).

5. W. B. Hutchinson, T. W. Watson and I. L. Dillamore, Iron Steel Inst. 207, 1479 (1969).

6. I. L. Dillamore, C. J. E. Smith and T. W. Watson, Met. Sci.J. 1, 49 (1967).

7. W. F. Hosford, Trans. Metall. Soc. AIME 230, 12 (1964). 